\title{
Identification of a likely pathogenic variant of $Y Y 1$ in a patient with developmental delay
}

\author{
Soyoung Bae ${ }^{1 \oplus}$, Aram Yang ${ }^{2 \oplus}$, Ja-Hye Ahn ${ }^{1 \oplus}$, Jinsup Kim ${ }^{1 *} * \oplus$, and Hyun Kyung Park ${ }^{1 \oplus}$ \\ ${ }^{1}$ Department of Pediatrics, Hanyang University Medical Center, Hanyang University College of Medicine, Seoul, Korea \\ ${ }^{2}$ Department of Pediatrics, Kangbuk Samsung Medical Center, Sungkyunkwan University School of Medicine, Seoul, Korea
}

Gabriel-de Vries syndrome, caused by the mutation of $Y Y 1$, is a newly defined genetic syndrome characterized by developmental delay, facial dysmorphism, and intrauterine growth retardation. A 7-month-old girl presented developmental delay and subtle facial dysmorphism including facial asymmetry, micrognathia, and low-set ears. Whole exome sequencing identified a de novo heterozygous missense variant in the $Y Y 1$ (c.1220A>G; p.His407Arg) gene. Here, we examined the clinical and genetic characteristics of an infant with a novel likely pathogenic variant of $Y Y 1$. This case expands the phenotypic spectrum of Gabriel-de Vries syndrome.

Key words: YY1 transcription factor, Developmental disabilities, Facial dysmorphism.

\section{Introduction}

Gabriel-de Vries syndrome (GADEVS, MIM \#617557) is a newly defined genetic syndrome characterized by developmental delay, craniofacial dysmorphism, intrauterine growth retardation, and other neurologic manifestations $[1,2]$. This neurodevelopmental disorder resulted from an aberration of the YY1 gene (MIM \#600013) located on chromosome 14q32.2, which encodes the Yin Yang 1 protein that plays a crucial role in neuronal development and neuroendocrine malignancy [3-5]. YY1 is a ubiquitous multifunctional transcription factor that activates the neuregulin-dependent peripheral nerve myelination, represses the transcriptional co-regulator Host Cell Factor $\mathrm{C}_{1}$, and maintains the proliferation and survival of neural progenitor cells at early developmental stages of brain [6-8].

Because next generation sequencing techniques, including chromosomal microarray and whole exome sequencing (WES), enables successful identification of various genomic variants in undiagnosed syndromic cases with developmental delay in a time- and cost-effective manner, the importance of exome sequencing for identifying the genetic etiology is gradually being emphasized $[9,10]$. Based on the data from multiple studies and the Deciphering Developmental Disorders Study database, more than 100 sequence and copy number variants of YY1 have been identified among patients with syndromic developmental delay $[1,2,11-14]$. Here, we report on a novel missense variant of $Y Y 1$, identified by WES, in an infant with a developmental delay. Owing to early diagnosis of GADEVS at infancy, it was easier for the patient to access intensive rehabilitation therapy that improved her cognitive outcome. To the best of our knowledge, this is the first report of GADEVS in the Korean population, which can help expand the clinical and molecular spectra of GADEVS.

Received: 1 June 2021, Revised: 11 June 2021, Accepted: 13 June 2021, Published: 30 June 2021

*Corresponding author: Jinsup Kim, M.D. (iD https://orcid.org/0000-0002-9641-5192

Department of Pediatrics, Hanyang University Medical Center, Hanyang University College of Medicine, 222-1 Wangshimri-ro, Sungdong-gu, Seoul 04763, Korea.

Tel: +82-2-2290-8380, Fax: +82-2-2290-8385,E-mail: pinees@gmail.com

Conflict of interest: The authors declare that they do not have any conflicts of interest.

(c) This is an open-access article distributed under the terms of the Creative Commons Attribution Non-Commercial License (http://creativecommons.org/licenses/by-nc/4.0/) which permits unrestricted non-commercial use, distribution, and reproduction in any medium, provided the original work is properly cited. 


\section{Case}

\section{Methods}

1) Whole exome sequencing and Sanger sequencing

This study was approved by the Institutional Review Board (IRB) of Hanyang University Medical Center, Seoul, Korea (IRB No. 2019-11-040). Informed consent was obtained from both parents of the participant. WES was performed in 3billion Inc. (Seoul, Korea) using genomic DNA isolated from the patient's whole blood. All exons from the entire human genome (approximately 22,000) were captured using an xGen Exome Research Panel v2 (Integrated DNA Technologies Inc., Coralville, IA, USA). The captured genomic regions were sequenced using a NovaSeq 6000 (Illumina Inc., San Diego, CA, USA). Raw genome sequencing data analyses included alignment to the reference sequence (National Center for Biotechnology Information genome assembly GRCh37; accessed in February of 2009). The mean depth of coverage was 100 -fold with $99.2 \%$ coverage. Variant calling, annotation, and prioritization were performed as previously described [15]. Developmental delay and facial dysmorphism served as filtering indices for clinical symptoms to analyze the candidate variants.

\section{Case report and result}

A 7-month-old girl visited the outpatient clinic for children because of developmental delay. The patient was born at 39+5 gestational weeks with a body weight of 3,140 g (-0.15 standard deviation [SD]) at birth. She was the only child of nonconsanguineous, healthy Korean parents with no family history of developmental delays or multiple congenital abnormalities. Prenatal examinations did not reveal any abnormalities. She presented a mild dysmorphic facial appearance including facial asymmetry, micrognathia, and low-set ears. The girl's height was $71.2 \mathrm{~cm}$ (1.7 SD), weight was $8.5 \mathrm{~kg}$ (0.9 SD), and head circumference was $43 \mathrm{~cm}(0.2 \mathrm{SD})$. She showed no apparent neurological abnormalities, such as change in muscular tone, increased deep tendon reflexes, or pathologic reflexes. Results of laboratory tests, including blood chemistry and endocrine tests as well as the results of tandem mass spectrometry screening of inborn errors of metabolism were normal. Results of abdominal ultrasound, echocardiography, skeletal survey, and brain magnetic resonance imaging at the age of 9 months were also normal. Audiometry revealed a mild sensorineural hearing loss in the right ear. An assessment using the Bayley Scales of Infant and Toddler Development 3rd edition (Bayley-III) at the age of
7 months demonstrated a mild degree of global developmental delay, especially in gross motor function.

To explore the possibility of a genetic syndrome, we performed genetic tests, such as chromosomal microarray and WES, to identify the underlying genetic defect. Chromosomal microarray revealed a normal karyotype of $46, X X$, without copy number variation. WES identified a novel heterozygous missense variant of c. 1220C >T (p.His407Arg) in the YY1 gene (Fig. 1). The parents did not have this variant (Fig. 1). This missense variant was validated by Sanger sequencing and was expected to damage protein structure, function, or protein-protein interaction (Table 1) [16]. This variant has not been reported in a large population cohort (The Genome Aggregation Database, the Exome Aggregation Consortium, 1000 Genomes, and the Exome Sequencing Project) and was classified as "likely pathogenic" by the American College of Medical Genetics and Genomics/Association for Molecular Pathology guidelines [17].

Currently, the patient is undergoing various rehabilitation treatments twice a week, such as occupational therapy, neurodevelopment treatment, and activities of daily living.

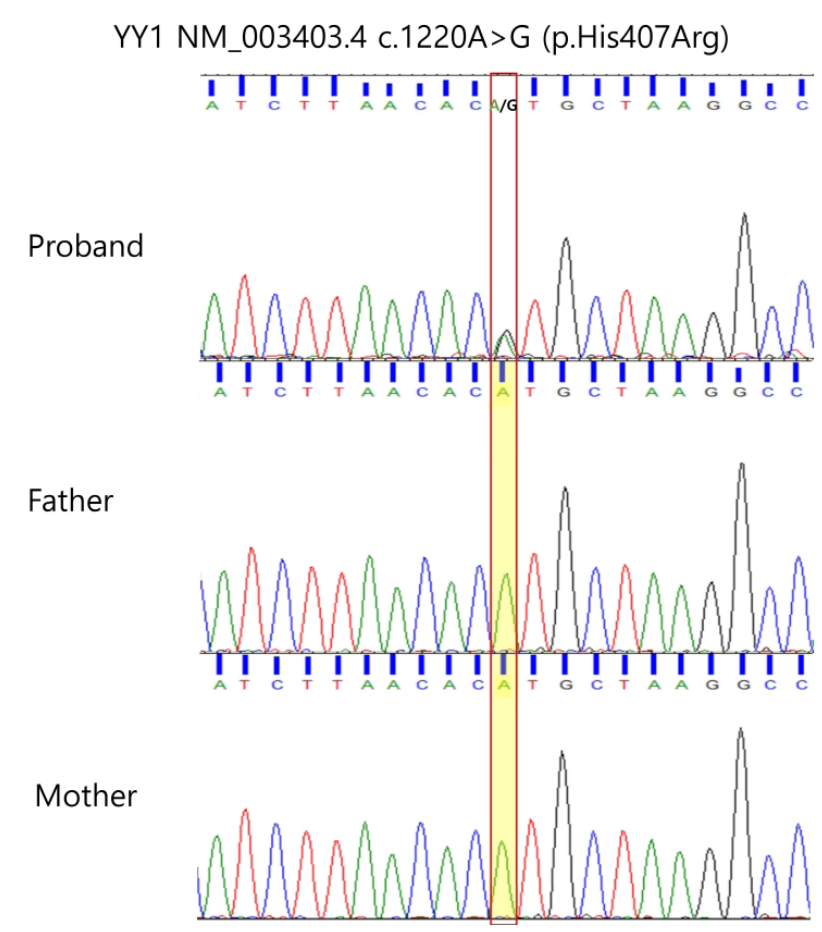

Fig. 1. Sanger sequencing of the proband and her parents. Sequencing showed a de novo novel pathogenic variant of the $Y Y 1$ gene (c.1220A>G; p.His407Arg). 
Table 1. Details of the variant identified in the $Y Y 1$ gene

\begin{tabular}{|lc|}
\hline \multicolumn{1}{|c}{ Information } & Variant \\
\hline Chromosome & 14 \\
\hline Position & 100743912 \\
\hline Reference & $\mathrm{A}$ \\
\hline Alteration & $\mathrm{G}$ \\
\hline GnomAD & Absent \\
\hline Nucleotide change & c.1220A $>\mathrm{G}$ \\
\hline Protein change & p.His407Arg \\
\hline Variant type & Missense variant \\
\hline Zygosity & Heterozygous \\
\hline REVEL score & ${ }^{\mathrm{a}}$ \\
\hline Classification $^{\mathrm{b}}$ & 0.847 \\
\hline
\end{tabular}

${ }^{2}$ The pathogenicity of missense variant was predicted using rare exome variant ensemble learner (REVEL) method. 'Variant classification is based on the American College of Medical Genetics and Genomics/Association for Molecular Pathology guidelines.

GnomAD, The Genome Aggregation Database; REVEL, rare exome variant ensemble learner.

Data from the article of loannidis NM et al. (Am J Hum Genet 2016;99: 877-85) [16]

\section{Discussion}

GADEVS, also known as YY1 haploinsufficiency syndrome, was first reported in 2017 by Gabriele et al. [1]. Since YY1 was considered a candidate gene for syndromic intellectual disability with intrauterine growth retardation and dysmorphic facial appearance on the basis of trio exome sequencing conducted by Vissers et al., 26 cases have been reported $[1,2,11-13]$. YY1 is a zinc finger containing multifunctional transcription factor that regulates transcriptional activation and repression in nervous systems [3]. Loss of function of YY1, which is caused by deletion, protein-truncating, or missense mutation affects, neural development, neuronal function, and developmental myelination $[1,3,18]$. YY1 was also involved in the tumorigenesis of various tumors mediated via p53 downregulation and may serve as a marker for diagnosis, treatment, and prognosis [5]. Furthermore, the targets differentially regulated by $Y Y 1$ include several genes associated with neurodevelopmental disorders, such as GTF2l, KANSL1, NRXN2, MED12, NSD1, ZBTB20, and HCFC1 [1,7]. Genotypes of GADEVS include five truncating mutations, eight mutations resulting in a missense change, or 13 deletions of $Y Y_{1}$ and other neighboring genes $[1,2,12,13]$. In the current study, the missense variant is located in the ultimate exon, where most of the pathogenic variants have been described $[1,13]$.

Phenotypical features of GADEVS include cognitive impairment, behavioral alteration, intrauterine growth restriction, feeding problems, various congenital malformations, and move- ment disorders [1]. Developmental delay was found in both motor function and speech, and a mild to profound degree of intellectual disability was presented in patients with pathogenic YY1 variant. Characteristic dysmorphic appearances were facial asymmetry, broad forehead, posteriorly rotated ears, periorbital fullness, bulbous nasal tip, and malar flattening. Here, the infant had shown mild developmental delay with subtle facial dysmorphism of facial asymmetry, micrognathia, and low set ears. Thus far, she has not shown feeding difficulties or other neurologic symptoms. However, regular evaluation of neurologic disorders is required because many neurologic symptoms, including movement disorders, are presented by patients aged $>10$ years $[1,2]$.

We identified a de novo missense variant of YY1, which was classified to be likely pathogenic, in an infant with mild developmental delay and subtle facial dysmorphism. Through this case, we expect to expand the clinical and genetic spectra of GADEVS that might be an unidentified cause of syndromic features with developmental delay and facial dysmorphism.

\section{Acknowledgements}

This work was supported by Hanyang Medicine-EngineeringBiology Center. The analysis of whole exome sequencing was supported by 3billion Inc. (Seoul, Korea).

\section{Authors' Contributions}

Conception and design: JK. Acquisition of data: SB, JHA, HKP, $J K$. Analysis and interpretation of data: $\mathrm{SB}_{1} \mathrm{JK}$. Drafting the article: $S B, A Y, J K$. Critical revision of the article: $S B, A Y, J K$. Final approval of the version to be published: All authors.

\section{References}

1. Gabriele $M$, Vulto-van Silfhout $A T$, Germain $P L$, Vitriolo $A$, Kumar $R$, Douglas $E_{1}$ et al. $Y Y 1$ haploinsufficiency causes an intellectual disability syndrome featuring transcriptional and chromatin dysfunction. Am J Hum Genet 2017;100:907-25.

2. Carminho-Rodrigues MT, Steel D, Sousa SB, Brandt G, Guipponi M, Laurent $\mathrm{S}$, et al. Complex movement disorder in a patient with heterozygous YY1 mutation (Gabriele-de Vries syndrome). Am J Med Genet A 2020;182:2129-32.

3. He $Y$, Casaccia-Bonnefil P. The Yin and Yang of $Y Y 1$ in the nervous system. J Neurochem 2008;106:1493-502. 
4. Cromer MK, Choi M, Nelson-Williams C, Fonseca AL, Kunstman JW, Korah RM, et al. Neomorphic effects of recurrent somatic mutations in Yin Yang 1 in insulin-producing adenomas. Proc Natl Acad Sci U S A 2015;112:4062-7.

5. Gordon S, Akopyan G, Garban H, Bonavida B. Transcription factor YY1: structure, function, and therapeutic implications in cancer biology. Oncogene 2006;25:1125-42.

6. He Y, Kim JY, Dupree J, Tewari A, Melendez-Vasquez C, Svaren J, et al. Yy1 as a molecular link between neuregulin and transcriptional modulation of peripheral myelination. Nat Neurosci 2010;13:147280.

7. Jolly LA, Nguyen LS, Domingo D, Sun $Y$, Barry $S$, Hancarova $M$, et al. HCFC1 loss-of-function mutations disrupt neuronal and neural progenitor cells of the developing brain. Hum Mol Genet 2015;24:333547.

8. Zurkirchen L, Varum S, Giger S, Klug A, Häusel J, Bossart R, et al. Yin Yang 1 sustains biosynthetic demands during brain development in a stage-specific manner. Nat Commun 2019;10:2192.

9. Battaglia A, Doccini V, Bernardini L, Novelli A, Loddo S, Capalbo A, et al. Confirmation of chromosomal microarray as a first-tier clinical diagnostic test for individuals with developmental delay, intellectual disability, autism spectrum disorders and dysmorphic features. Eur J Paediatr Neurol 2013;17:589-99.

10. Srivastava S, Love-Nichols JA, Dies KA, Ledbetter DH, Martin CL, Chung WK, et al.; NDD Exome Scoping Review Work Group. Metaanalysis and multidisciplinary consensus statement: exome sequencing is a first-tier clinical diagnostic test for individuals with neurodevelopmental disorders. Genet Med 2019;21:2413-21.
11. Vissers $L E$, de Ligt J, Gilissen $C$, Janssen I, Steehouwer M, de Vries $P$, et al. A de novo paradigm for mental retardation. Nat Genet 2010;42:1109-12.

12. Morales-Rosado JA, Kaiwar C, Smith BE, Klee EW, Dhamija R. A case of YY1-associated syndromic learning disability or Gabrielede Vries syndrome with myasthenia gravis. Am J Med Genet A 2018;176:2846-9.

13. Tan L, Li Y, Liu F, Huang Y, Luo S, Zhao P, et al. A 9-month-old Chinese patient with Gabriele-de Vries syndrome due to novel germline mutation in the YY1 gene. Mol Genet Genomic Med 2021;9:e1582.

14. Firth HV, Richards SM, Bevan AP, Clayton S, Corpas M, Rajan D, et al. DECIPHER: database of chromosomal imbalance and phenotype in humans using ensembl resources. Am J Hum Genet 2009;84:524-33.

15. Seo GH, Kim T, Choi IH, Park JY, Lee J, Kim S, et al. Diagnostic yield and clinical utility of whole exome sequencing using an automated variant prioritization system, EVIDENCE. Clin Genet 2020;98:562-70.

16. Ioannidis NM, Rothstein JH, Pejaver V, Middha S, McDonnell SK, Baheti $S$, et al. REVEL: an ensemble method for predicting the pathogenicity of rare missense variants. Am J Hum Genet 2016;99:877-85.

17. Richards S, Aziz N, Bale S, Bick D, Das S, Gastier-Foster J, et al.; ACMG Laboratory Quality Assurance Committee. Standards and guidelines for the interpretation of sequence variants: a joint consensus recommendation of the American College of Medical Genetics and Genomics and the Association for Molecular Pathology. Genet Med 2015;17:405-24.

18. Donohoe ME, Zhang X, McGinnis L, Biggers J, Li E, Shi Y. Targeted disruption of mouse Yin Yang 1 transcription factor results in periimplantation lethality. Mol Cell Biol 1999;19:7237-44. 